\title{
Dynamic Simulation Analysis of the Mid-Strain-Rate Tensile Testing Machine
}

\author{
Xinyu Feng ${ }^{\text {a }}$, Yonggang Gu ${ }^{\text {b }}$, Huijuan Wang ${ }^{c}$, Chao Zhaid \\ School of Engineering Science, University of Science and Technology of China, Hefei \\ 230031,P.R.China \\ afxinyulf@mail.ustc.edu.cn, byggu@ustc.edu.cn, wanghuij@ustc.edu.cn, dzhaichao@ustc.edu.cn
}

Keywords: Mid-strain-rate, Collision, Deformation analysis, Modal analy.

\begin{abstract}
In order to test the mid-strain-rate, a specialized fixture that can be installed on the MTS809 test machine is designed. A three-dimensional solid model of the fixture was established with SolidWorks, and multi body dynamics simulation analysis on the tensile process of dumbbell shaped flat specimens was carried out in the strain rate range of $1 \mathrm{~s}^{-1} \sim 500 \mathrm{~s}^{-1}$ by in ADAMS, then the collision cushioning impact pad block and an inner end cover of the contact force, the deformation velocity and deformation under given constraint condition and stretching velocity were obtained. Finite element analysis is carried out on the main components of the impact force by Workbench ANSYS basing on the conclusions obtained from ADAMS, then the deformation characteristics and stress distribution of impact block, inner end cap and fastening screw are obtained and the modal analysis of the whole fixture is carried out. Results indicate that 1.the fixture meets the design requirements and is able to conduct the whole acceleration - collision - deceleration - uniform tension test procedure, and transfer the kinetic energy to the tester; 2.the maximum stress and deformation of the important parts are all in the range of the material, thus no plastic deformation occurs; 3.there's no resonance in the stretching process.
\end{abstract}

\section{Introduction}

The mid-strain-rate is the transition zone in which the material properties' sensibility to strain rate increases from non-obvious to obvious, so it is necessary to explore the strain rate dependence of the strain rate zone in the material ${ }^{[1]}$. Scholars from all over the world have done some mid-strain-rate test on different materials. For example, LI Xibing and LUO Zhang ${ }^{[2]}$ studied the tensile behavior of steel fiber reinforced concrete under medium strain rate with a self designed dynamic testing system. WU Hengyi and MA Gang ${ }^{[3]}$ researched PMMA's uniaxial tension behavior under low and mid-strain-rate on MTS810 testing machine and self-developed strain rate testing machine. E.H. Wong $^{[4]}$ tested stress - strain characteristics of tin-based solder alloys under medium strain rate.

In this paper, the strain rate and tensile properties of materials are obtained on MTS809 tensile testing machine. Unlike quasi-static tensile tests, mid-strain-rate tensile tests require the tensile section to be loaded at a certain speed before pulling the specimen. In this process, the tensile end will collide with the inner end cover. The impact pad driven by a piston rod, hits the inner cover and causes considerable impact force, which might cause part deformation. Therefore, it is necessary to carry out quantitative simulation of the fixture to determine whether it can work properly.

ADAMS is appropriate for system motion and dynamics analysis, but it is not suitable for finite element analysis; while ANSYS is appropriate for linear/ non-linear stress, strain analysis ${ }^{[5]}$. Thus in this paper, simulation of the fixture's overall motion is first conducted in ADAMS to obtain the contact force at the collision moment, which is then transfered into ANSYS to carry out stress, deformation analysis and modal analysis of important parts. Through the joint simulation analysis between ADAMS and ANSYS software, the performance of the fixture is predicted more accurately in the design stage. The obtained data provides the basis for test machine development and parameter optimization. 


\section{Fixture Overall Working Process}

As shown in Figure 1, the fixture is fixed on the MTS809 testing machine base by eight screws. The bottom of specimen is tighten by two clips and a taper sleeve, the top of the specimen was caught on the test machine. The impact piston at the bottom travers an acceleration distance of $50 \mathrm{~mm}$ under piston rod's drive, and then hits the inner cap. The impact pad is made by PTFE to reduce the impact of shock. The whole process is accelerated - collision - deceleration - uniform tensile test specimen. The material properties of each part are shown in Table 1.

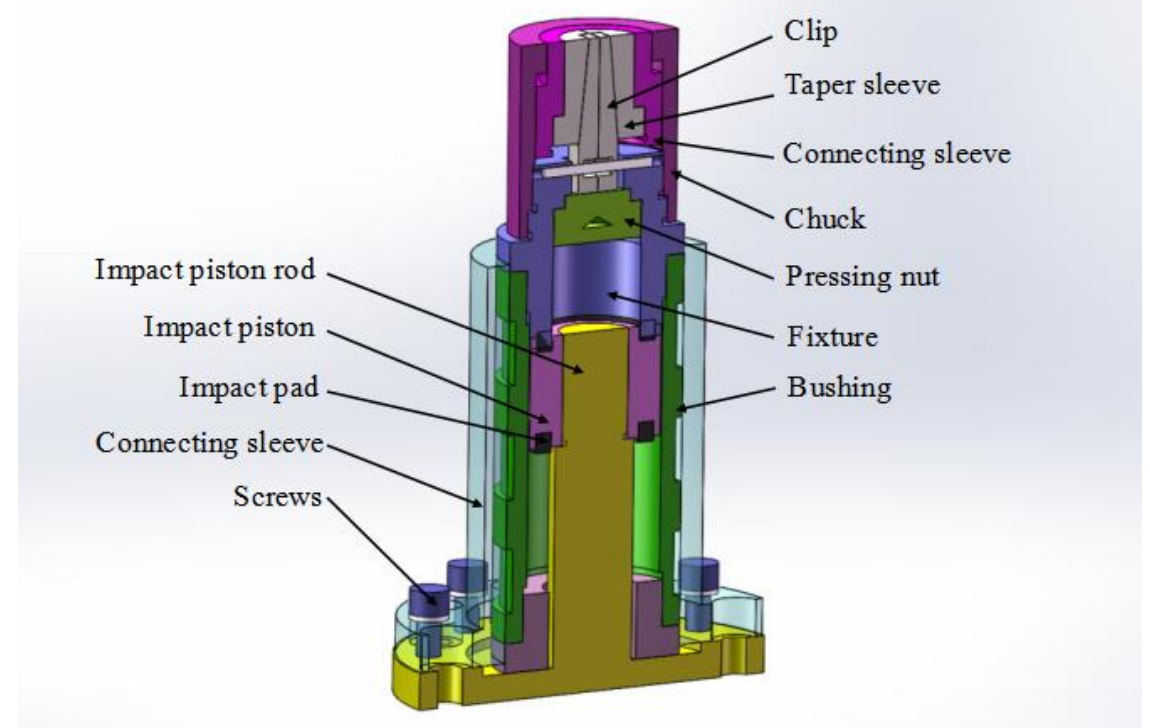

Fig. 1 Fixture whole

Table 1. Part material setting

\begin{tabular}{ccccc}
\hline Components & Material & $\begin{array}{c}\text { Young's } \\
\text { Modulus } \\
(\mathrm{N} / \mathrm{m} 2)\end{array}$ & $\begin{array}{c}\text { Density } \\
(\mathrm{kg} / \mathrm{m} 3)\end{array}$ & Poisson's Ratio \\
\hline Impact pad & PTFE & $2.8 \mathrm{E}+8$ & 2150 & 0.4 \\
Inner cap & $\mathrm{Cr} 12 \mathrm{MoV}$ & $2.18 \mathrm{E}+11$ & 7850 & 0.28 \\
Connecting sleeve & $\mathrm{ZQSn6}-6-3$ & $2.06 \mathrm{E}+11$ & 8820 & 0.3 \\
Other parts & $40 \mathrm{Cr}$ & $2.11 \mathrm{E}+11$ & 7870 & 0.277 \\
\hline
\end{tabular}

\section{Fixture Simulation Based on ADAMS Software}

Collision interfaces between some parts are complex surface, thus the fixture requires to be designed to ensure smooth energy transmission and coordination of motion between different parts. The collision moment is simulated in ADAMS to observe the energy transfer situation, which is significant for the overall design of the test machine.

Although ADAMS / View provides a complete modeling function, CAE software are more appropriate for modeling parts with complex shape. Thus the model is built in SolidWorks and then transferred into ADAMS, as shown in Figure 2. The dumbbell shaped flat specimen is replaced by a flexible connecting spring because tension process of rigid bodies cannot be simulated in ADAMS. 


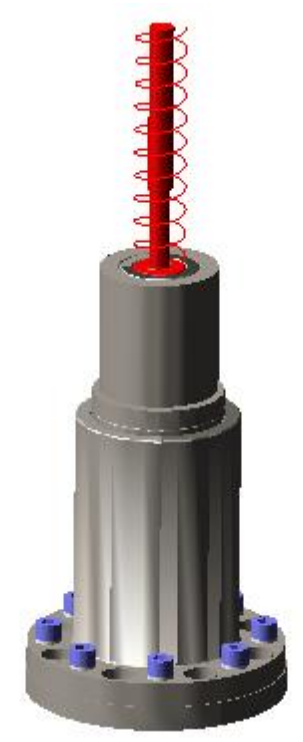

Fig. 2 Fixture model in ADAMS

\subsection{Theoretical Calculation of Collision Process}

The collision process belongs to the process of load deformation and nonlinear dynamic contact, which usually lasts $0.1 \sim 0.2 \mathrm{~s}$, and the kinetic energy is rapidly converted into other forms of energy.

Contact constraints ${ }^{[6]}$ between the pad and the inner cover are added to prevent penetration phenomenon during collision simulation. When the fixture is in operation, the contact between the impact pad and the inner cap is continuous. The ADAMS system defines this contact as a non-linear spring, and the Young's Modulus of the part material is equivalent to the stiffness of the spring, Damping is equivalent to energy loss. In the method of defining the contact force in ADAMS / Solver, the most widely used impact ${ }^{[7]}$ of the impact function method is that the contact force is actually equivalent to the force produced by a spring damper. Impact function model to obtain the normal contact force $F_{n}$ formula is as follows:

$\mathrm{F}_{\mathrm{n}}=\mathrm{k} \cdot \mathrm{g}^{\mathrm{e}}+\mathrm{c} \cdot \mathrm{d}_{\mathrm{g}} / \mathrm{d}_{\mathrm{t}}$

Where $\mathrm{k}$ is the stiffness coefficient; $\mathrm{g}$ is the instantaneous penetration of two contact objects; e is the force index; $\mathrm{c}$ is the damping coefficient. Simulation and related post-processing could be conducted after contact settings are completed according to formula (1).

\subsection{Simulation and Results Analysis}

Add a downward translation motion to the translational joint on the impact piston rod, and set the movement speed with the STEP function. Set the simulation steps to 400, To

Prevent simulation failures and improve the time resolution of the results, the number of

Simulation steps should be taken as large as possible in the calculation of capacity.

After running the simulation to get contact when the impact of the pad and the inner

cap of the force curve, the maximum force of $28000 \mathrm{~N}$, the force of the flexible spring is substantially equal to the contact force, as shown in Fig. 3, and it is understood that the

Energy loss is negligible in the transmission.

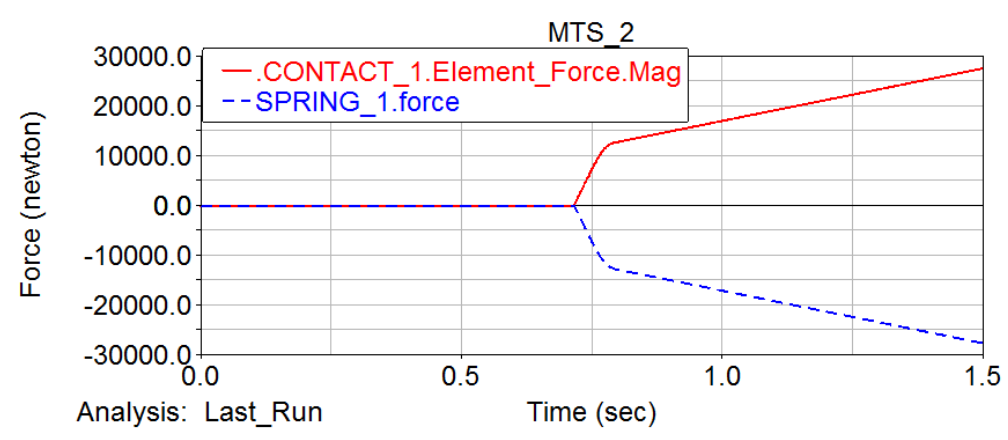

Fig. 3 Comparison of the force between the contact and the specimen 


\section{Static Simulation Analysis of Fixture}

Here we use ANSYS / Workbench's contact analysis model and modal analysis model.

\section{1 mpact Simulation of Inner Cap and Impact Pad}

Import the inner cap and the impact pad at the moment of the impact of the three-dimensional model. This structure is relatively simple, so automatically partition method in ANSYS/Workbench is selected to mesh the model obtained. 139893 nodes and 92554 unit are generated in this mesh. Meshing model diagram is shown in Figure 4:

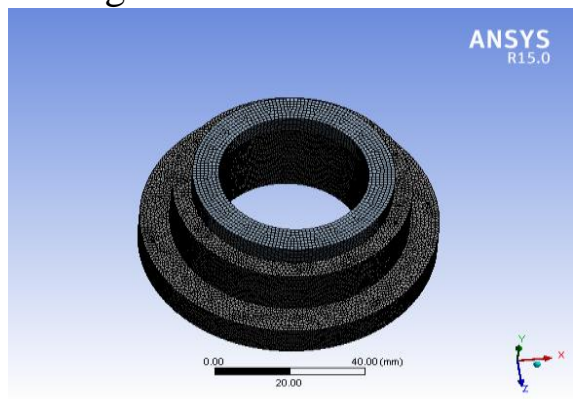

Fig. 4 Meshing of the inner end cap and impact lining

The simulated contact force is $28000 \mathrm{~N}$. Therefore, the same interaction load is applied to the inner and outer hoods, and the rigid restraints are applied to the C, D, E, And F surfaces. Due to the greater pressure generated by the collision, the maximum stress and deformation of the inner cap and the impact pad is the key to the design. Figure 5 is deformation nephogram of contact analysis, Figure 6 is stress nephogram of contact analysis. From the two figures we can know that the maximum deformation of the inner cap in $7.5083 \times 10-5 \mathrm{~mm}$ or less, which could be ignored. The maximum deformation is $0.177 \mathrm{~mm}$ and occurs in the cushion impact pad. The maximum stress is $7.05 \mathrm{MPa}$ and also occurs in the impact pad, which is much lower than the compressive strength of PTFE (19.52MPa under $10 \%$ deformation.

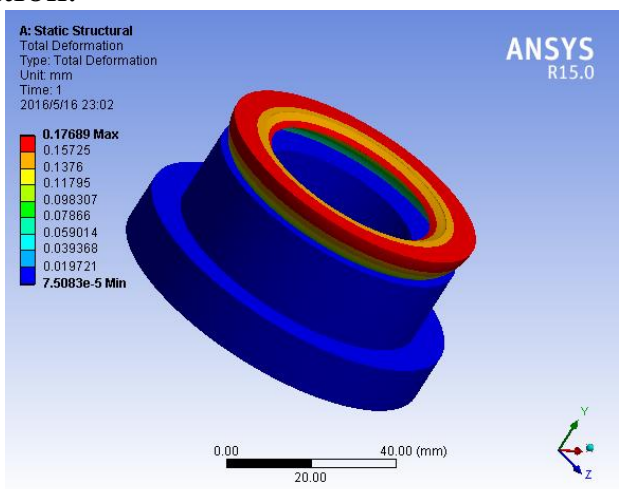

Fig. 5 Deformation nephogram of contact analysis

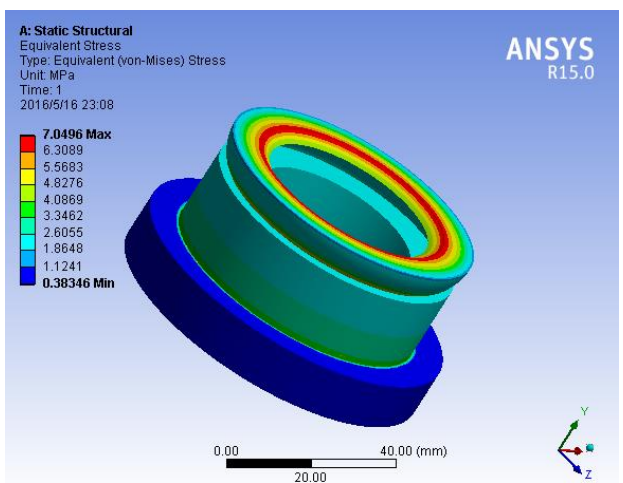

Fig. 6 Stress nephogram of contact analysis 


\subsection{Analysis and Simulation of Impact Piston Rod, Connecting Sleeve and Cylindrical Head Screws}

Collision occurs in the tension process. Thus standard parts also requires to be simulated to verify if their deformation and stress to meet the requirements in the mechanical performance. The entire fixture is fixed by eight M10×30 studs on the base, and the connecting sleeve and impact piston rod are more impacted on the piston rod and the connecting sleeve, therefore, the meshing method is selected by multi-domain swept grid. 19642 nodes and 3408 units are generated.

The overall load applied downward load of 30000 N. Figure 7 shows the connection sleeve and screw deformation diagram, as shown in Fig.7 the maximum deformation occurred in the screw and the connecting sleeve contact surface, deformation in the $2.19 \times 10-4 \mathrm{~mm}$ or less. Figure 8 is the stress map, since the impact of the piston rod is not subject to the stress, so it is hidden in the figure. Obviously, the maximum stress appears in the screw and connecting sleeve connection, the maximum stress is $20.02 \mathrm{MPa}$, which is much lower than the allowable stress (160MPa).

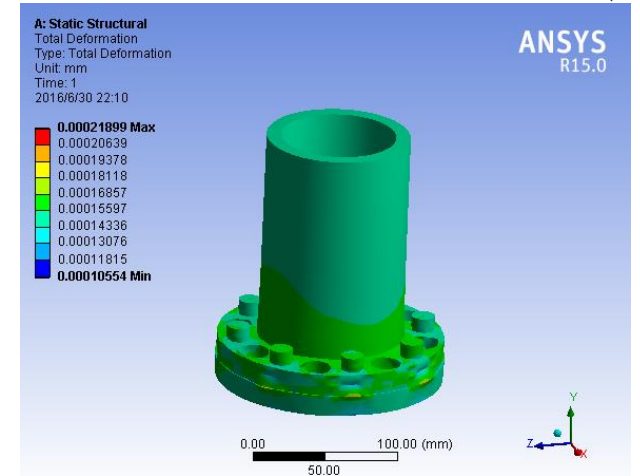

Fig. 7 Deformation nephogram of impact piston rod, connecting sleeve and screw

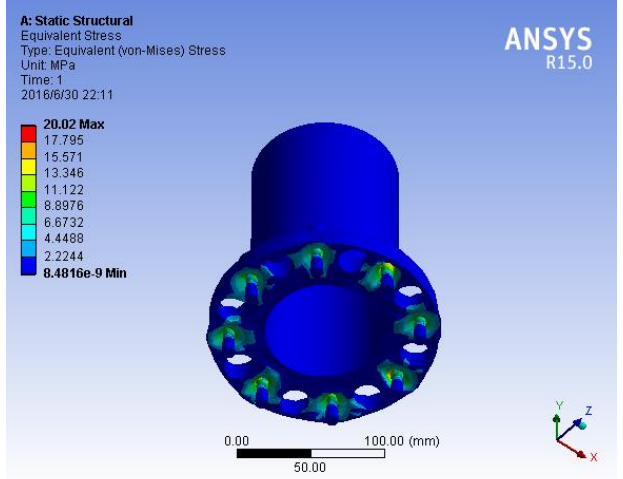

Fig. 8 Stress nephogram of impact piston rod, connecting sleeve and screw

\subsection{Modal Analysis}

The natural frequencies of the structure and the corresponding shape of the modal structure are important parameters for the design of structures subjected to variable load conditions. Therefore, modal analysis is necessary. In the ANSYS Workbench, it is assumed that ${ }^{[8]}$ : (1) the damping effect is not taken into account unless the damping feature solution is specified; (2) the structural stiffness matrix and the mass matrix do not change; (3) the load is constant.

The free vibration equation in the undamped system ${ }^{[9]}$ is shown below:

$[\mathrm{M}]\{\ddot{u}\}+[\mathrm{k}]\{\mathrm{u}\}=\{0\}$

Where $[\mathrm{M}]$ is mass matrix; $[\mathrm{k}]$ is stiffness matrices; $\{\ddot{u}\}$ is node acceleration

Vector; $\{\mathrm{u}\}$ is nodal displacement vector;

Main vibration:

$\{\mathrm{u}\}=\{\Phi\}$ Ta $\sin (\omega \mathrm{t}+\varphi)$

The necessary and sufficient condition that $\{\Phi\}$ has a nonzero solution is:

$|[\mathrm{K}]-\omega 2[\mathrm{M}]|=0$

The solutions are ascending sorted: 
$0<\omega 21 \leq \omega 22 \leq \ldots \leq \omega 2 n$

$\omega 1$ is fundamental frequency; $\omega i$ is the ith order natural frequency.

Small features such as keys, pins, fillets, chamfers, etc. increase the time and difficulty of modal analysis, so these features are simplified in modeling. The material is set as shown

In Table 1 above. The overall structure of the fixture is irregular, and the different parts can be divided into different parts in the Workbench. We choose to clamp the clip, the Compression nuts and the connecting sleeve for automatic meshing, and other parts are divided into multi regional grids. The results of mesh division are shown in figure 9, and the number of nodes is 156663 and the number of units is 57116

According to the actual situation, the impact piston rod bottom has eight threaded holes, it is fixed on the machine by $8 \Phi 13$ screw, so the simulation of 8 holes with fixed Support, constraint the holes of all degrees of freedom. The modal order is 6 , and the first 6 natural frequencies are shown in Table 2 , and the vibration modes are shown in figure 10 15. The displacement change in the vibration pattern is a relative value, so it does not represent the actual physical displacement, but only reflects the proportion of deformation indifferent parts [10].

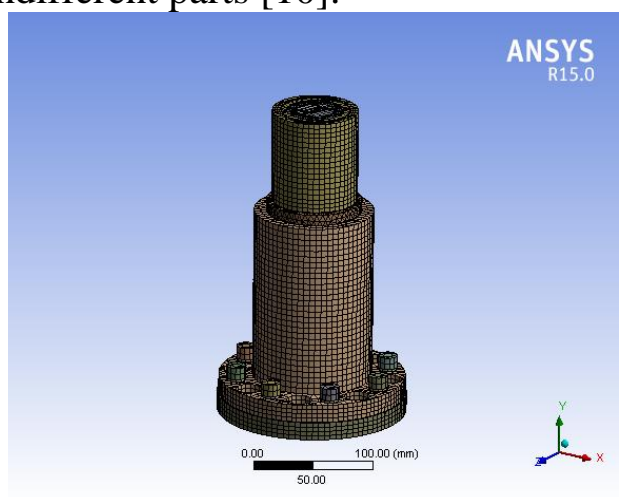

Fig. 9 overall meshing of fixture

Table 2. The first 6 natural frequencies of the fixture

\begin{tabular}{ccc}
\hline Order & Frequency $(\mathrm{Hz})$ & Mode description \\
\hline 1 & 712.67 & fixture swings left and right \\
2 & 714.23 & fixture swings back and forth \\
3 & 2084.6 & along the spindle expansion contraction state \\
4 & 2316.7 & spindle vibration up and down \\
5 & 3060.4 & fixture left and right twist \\
6 & 3068 & fixture twists and turns \\
\hline
\end{tabular}

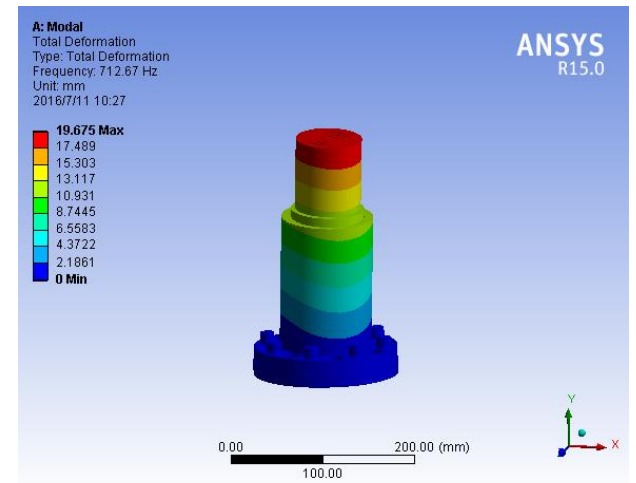

Fig. 10 NO.1 mode shapes 


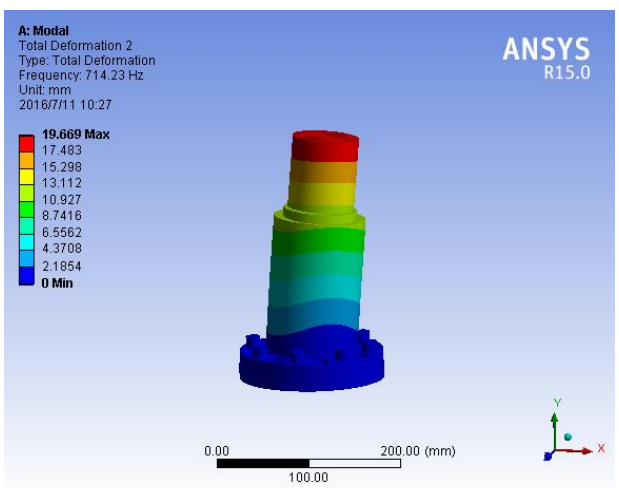

Fig. 11 NO.2 mode shapes

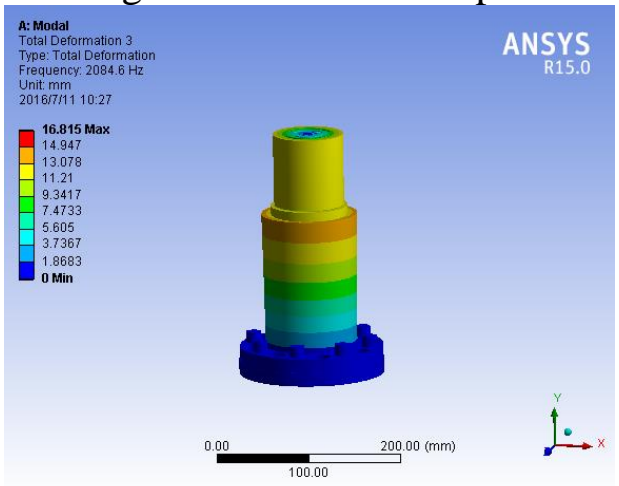

Fig. 12 NO.3 mode shapes

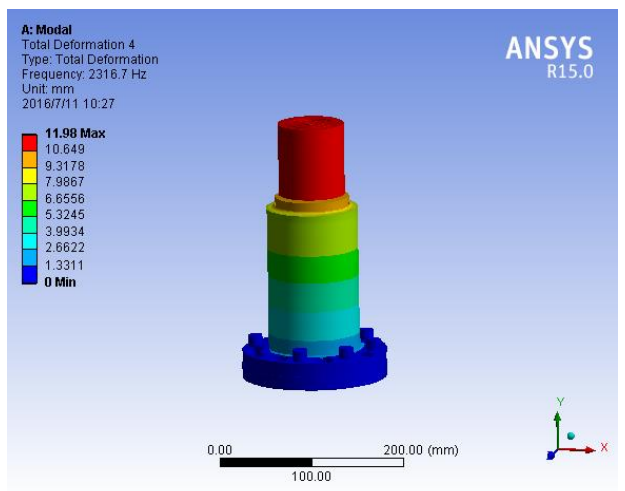

Fig. 13 NO.4 mode shapes

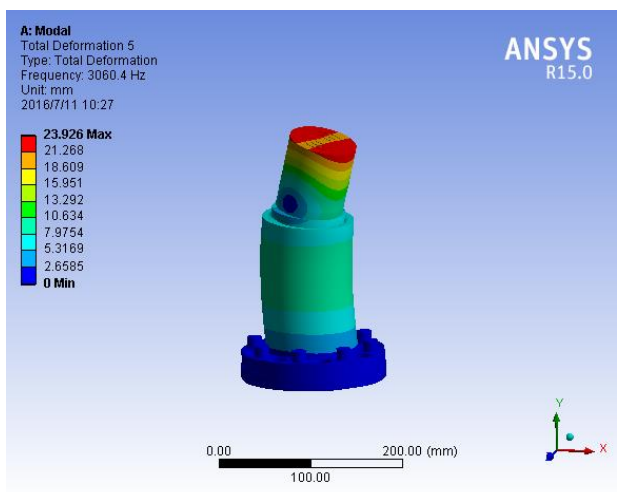

Fig. 14 NO.5 mode shapes 


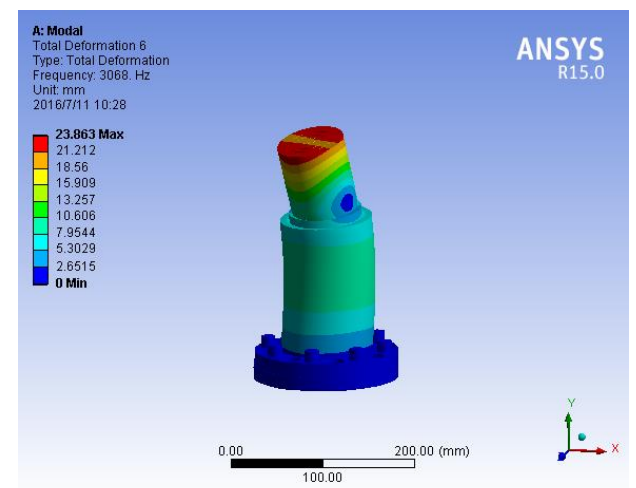

Fig. 15 NO.6 mode shapes

It is observed from the 6 order modal frequency and vibration modes that the natural frequency is very high and there are similar vibration modes under different phase because the fixture is axial symmetry [11]. The modal frequencies of the first two steps should be designed as far as possible to the operation frequency range to avoid resonance response and reduce structural damage of the test machine.

\section{Conclusion}

(1) The force transmitted to specimen is basically equal to the collision force, so the designed fixture is able to conduct tension test.

(2) All the strain and stress are in tolerance range, so the static design is usable. The lower order natural frequency goes beyond the operation frequency range, so resonance vibration won't occur.

\section{References}

[1]. ZHANG Xuefeng, XIA Yuanming. Research of a mid-strain-rate material testing machine [J]. Journal of Experimental Mechanics, 2001, 16(1).

[2]. LI Xibing, LUO Zhang, ZHAO Fubing. Experimental research of tension process of SFRC under mid strain rate [J]. Journal of Experimental Mechanics, 2004, 19(3): 301-309.

[3]. WU Hengyi, MA Gang, XIA Yuanming. Experimental research of PMMA's uniaxial tension behavior under low and mid-strain-rate [J]. Journal of Experimental Mechanics. , 2005, 20(2): 193-199.

[4]. Wong E H, Selvanayagam C S, Seah S K W, et al. Stress-strain characteristics of tin-based solder alloys at medium strain rate [J]. Materials Letters, 2008, 62(17): 3031-3034.

[5]. HOU Hongling, ZHAO Yongqiang, WEI Weifeng. Kinetic Simulating and Analyzing Based on ADAMS and ANSYS [J]. Modern Machinery, 2005, 4: 62-63.

[6]. XIE Zuiwei, WU Xinyue. Simulation analysis of impact process based on ADAMS [J]. Proceedings of the 3rd China CAE Analysis Technology Annual Conference, 2007: 339-342.

[7]. LI Zenggang. Introduction of ADAMS with examples [M]. Bei Jing: National Defense Industry Press, 2014.

[8]. JIA Zhihong, YANG Xianqin. Research of automatic rifle's dynamic model based on impact simulation in ADAMS [J]. Science and Technology Innovation Herald, 2011 (25): 17-17.

[9]. ZHANG Yan. Introduction to finite element analysis based on ANSYS Workbench 15.0[M]. Bei Jin: China Mechine Press, 2014.9:73-75.

[10]. NI Zhenhua. Mechanics of vibration [M]. Xi'An: Xi'an JiaoTong University Press.1988.

[11]. Yan A, Yang L, Wang S, et al. Modal Analysis andResearch of Spherical Plain Bearings in Different Materials based on the ANSYS-Workbench[J]. Proceedings of International Conference on Industrial Technology and Management Science, 2015. 